\title{
An Efficient Route Failure Detection Mechanism with Energy Efficient Routing (EER) Protocol in MANET
}

\author{
D. S. Jayalakshmi ${ }^{1}$ \\ ${ }^{1}$ Department of Physics, Sathyabama Institute of science and technology, Chennai, India \\ E-mail: jayalakshmi.physics@ sathyabama.ac.in
}

\author{
D. Hemanand ${ }^{2} *$, G. Muthu Kumar ${ }^{3}$, M. Madhu Rani ${ }^{4}$ \\ ${ }^{2 *}$ Department of Computer Science and Engineering, Sriram Engineering College, Tiruvallur, India \\ ${ }^{3}$ Department of Computer Science and Engineering, Sriram Engineering College, Tiruvallur, India \\ ${ }^{4}$ Department of Information Technology, Rajalakshmi Engineering College, Chennai, India \\ E-mail: *d.hemanand@gmail.com, gmuthu79@gmail.com, madhurani.m@ rajalakshmi.edu.in
}

Received: 14 August 2020; Accepted: 13 December 2020; Published: 08 April 2021

\begin{abstract}
Mobile ad-hoc network (MANET) is a network with two or more number of nodes with restricted energy constraint. The high dynamic nature in MANET attracts needs to consider the energy efficient features in their construct. The routing protocol is an important criterion to be considered for evaluating the performance of the MANET. Energy consumption plays vital role in MANET. Hence designing the scheme that supports energy efficient is much needed for the high dynamic MANET environment concerned. This paper proposes the Energy Efficient Routing (EER) protocol based on efficient route failure detection. The Scope of this paper is to suggest a fresh routing procedure for Mobile Adhoc Network minimizes unsuccessful communication. The projected procedure practices three important criterions to locate the path that assure authentic communication. The channel caliber, connection caliber and node's residual energy is important reason for the failure of the node in MANET. Hence, the suggested routing mechanism believes these three different parameters to choose the finest node in the route. The reliable transmission and reception are attained by transferring information through route nominated by the suggested system verified by means of NS-2 simulator.
\end{abstract}

Index Terms: Channel caliber, Connection caliber, Energy level, Mobile Adhoc Network (MANET).

\section{Introduction}

Mobile Ad-hoc Network is an infrastructure less internet protocol centered system of wireless mobile points linked by microwaves. Connection points of a MANET have no unified management tool. It is popular because of its link stuffs where each connection point performance as a" router" to advancing the movement to additional listed points in the link. MANET attracts large responsiveness by variety uses on account of its exceptional characteristics mentioned here.

- Each portable connection point is an autonomous point, can function as both a host and a router. It is independent in behaviour.

- The connection points can link or move out the link always, creating the link configuration active in environment.

- Portable connection points are a smaller amount memory, power and other good features.

- Movable and unplanned manner loads smallest human involvement to setup the system or link.

- MANET is comfortable to design and install.

- The developed energy efficient route provides high performance of the network significantly. The higher longevity of the route provides high performance of the network in terms of packet delivery

- Throughput and make the network resources more useful. The developed stable route minimizes the link failure probability by exploiting the maximum residual

- Battery energy and network life time will be enhanced. 
In Armed forces field of battle [1] MANET is used to interchange the data between armed forces and automotive. MANET is predominantly applied for Meeting usage since it can provide the flash connection between cellular instruments for example tablet and notebook computers. Features like basic structure less and instant connection make MANET be applied simply for disaster situations.

The network topology in MANET is highly active due to the displacement of connection points; therefore, current term undergoes regular path breaks. This situation often leads to highly dynamic environment and minimize the QoS [2]. Then QoS is mandatory as MANET is predominantly applied for serious and delicate usage. The parameter of QoS are Data rate, Jitter and reliability. The disturbance between the nodes largely impact the QoS [3].

As MANET is a decentralized network, it lacks in admission control. The admission control is much important to decide whether the available resources is sufficient for reliable communication as it does not has fixed infrastructure. The multi-hop communication in MANET is severely affected by high speed variation in the quality of channel exists between the nodes. The link quality of the points in the multi-hop path may affect the QoS provided by MANET. The energy of the point in multi-hop path is also important to guarantee the reliable communication [4]. If the node energy level goes beyond the certain level, [5] it will not able to transmit the data instead it becomes dead node. Various protocols are available to select the relay nodes in multi-hop path. But they are not providing the complete solution for all above started problem in MANET.

The suggested EER method improves intelligence to nodes and develops a advanced route during the discovery phase. By taking into account, nodal energy and current queue status, network lifetime, throughput, end to end delay and improves network performance significantly.

\section{Literature Review}

The restricted assets in MANETs ready the designing an effective and consistent routing mechanism a very provocative complexity. A smart directing mechanism is essential to effectively consume the restricted assets all together be flexible to dynamic network environment circumstances for instance, traffic density, link extent and link splitting. Meanwhile, the routing mechanism should offer diverse levels of QoS to dissimilar applications. Proactive and Reactive are the two different classification of MANET. A proactive is also identified to as "table-driven" protocol. With a proactive, connection points in a MANETs consistently examine paths to all accessible nodes and try to keep reliable, updated direction-finding data. Hence, a starting point can catch a moving track instantly. It leads to high routing overhead. Reactive for MANETs are also known as on-demand routing protocols. In a reactive, routes are examined only need arises. A path finding process appeals a path-finding procedure. When a path has been initiated or no path is found after inspection for all path arrangements, then we can come to conclusion that path finding process gets completed. For extremely dynamic huge scale mobile ad hoc networks, proactive routing is not finest suit [6]. Beside we discuss further about recent routing mechanisms proposed by various authors in this section.

Zehua Wang et.al., [7] projected (PSR) Proactive Source Routing Protocol there by attained unscrupulous data passing conceivable in MANET. To establish the spanning tree between adjacent nodes, each connection point occasionally interchanges the data required. Hence, we came to understand that each connection point in the network is having route to access the other access points in the network. This routing mechanism permits source routing that minimizes the routing complexity. Then it will not believe the QoS measures. It directs to common route failure in the extremely unpredictable MANET environment.

Lavanya et. al., [8] investigated that connection loss is the important characteristic of putting down the operation of the network. They calculated the path failure of Ad hoc On-Demand Distance Vector (AODV) and suggested the answer to improve. A new method is suggested to reduce the path loss in network by gathering the substitute route. In this method, in-between connection points in the route involved in the path finding mechanism. So, overall complexity is minimized. Connection loss is only taken in to account for path loss in this approach.

In MANET Communication, the connection points can be compromised for the period of path finding mechanism. Ritu Sharma et.al., have selected the Ad-hoc On Demand Distance Vector (AODV) directing protocol because of its best performance in highly dynamic MANET to include security for the route. The AODV is modified to Proficient Adhoc On demand Distance Vector Routing (PASODV) [9] by using genetic algorithm [10] to discover the best route and to reconstruct the route in case of route failure. The Hash chain and digital signature algorithm are used to maintain the security in PAODV.

Michal Pioro et.al., [11] have observed the problem on the enhancements MANETs. The connections volume is changed on because of variation in the route. To address this trouble in this enhancement process, the connection readiness is taken in account for the path finding process. The authors built a model with path finding mechanism to minimize the connection rate trouble. He also briefly stated that route failure can happen because of the energy level of the in-between nodes.

Fan Bai et.al., [12] analyzed the impact of mobility in adhoc routing protocols in terms of statistics of path duration. From the analysis, the authors suggested that exponential distribution with suitable parameterization will give the accurate path duration for various mobility models. The capacity of the channel and the energy level is not considered for the estimation of path duration. 
Jihui Zhang et.al., [13] resolved the enhancement complexity in network routing by infer the active algorithm. The strong point of the route gets changed on because of the motion of the points in link.

Mahaboob et al., [14] suggested the attacks types for each network layer are identified and addressed to be prevented. The measures listed in this work are convertible as a modular component of any automated framework to make the complete attack prevention mechanism automated.

Mohammed Azza [15] proposed an enhanced approach based on first-hand reputation with allows to detected misbehavior node in MANET.

Tanweer Alam et al., [16] proposed a solution that describes the convergence of MANET in the IoT. The results found in this paper have been tested and implemented using different seniors.

Salem Satia et al., [17] aimed to evaluate how multiple hops influence the bandwidth and delay. The results show that OLSR performs better regarding the throughput. But Babel has less delay and faster regarding convergence.

Rafi et al. [18] proposed three critical issues encountered in Integrated Internet-MANET are addressed together. These issues are, efficient gateway selection mechanism, adjusting the gateway advertisement range and dynamically adapting the periodicity of gateway advertisements.

\subsection{Author Contribution}

In this paper, we suggest a novel energy efficient routing mechanism (EER) protocol. The suggested routing mechanism, investigate the channel volume. Suppose the volume is crosses the threshold limit, it will permit direct communication between the nodes. Or else multi hop path adopted. The parameters such as channel caliber, connection caliber and nodes' energy will decide selection of relay node in the multi hop. By means of these three measures during path finding action will predominantly minimize the path loss or route failure in the system. Here Proactive Source Routing Protocol (PSR) [7] is going to compare with our proposed model.

The left over sections of this paper is arranged as follows. In Section II, discussing the Existing works in detail and also linked to the proposed work is delivered. In Section III, we suggest the energy efficient authentic routing mechanism. Section IV discussing simulation results and at last, conclusion is drawn in Section V.

\section{Proposed Work}

An Ad-hoc is a gathering of portable points actively establish an impermanent link lacking application of some prevailing link resources or concentrated supervision. Mobile nodes are unrestricted to go casually then arrange itself randomly. Hence, wireless topology may change quickly and randomly. The extremely changing nature of a MANET leads regular then random variations of link structure, increasing trouble and complication to sending and receiving information in the middle of portable points. Contests and complications, joined by means of serious significance of directing mechanism in creating transportations between points. The multi-hop track is exposed by applying directing mechanisms in MANET.

Directing mechanisms prevailing for MANET must assure Quality of Service in the changing condition. Act of giving Quality of Service is a deadly process makes the MANET as highly dynamic. The consistent communication can be guaranteed by applying high QoS.

In a complete ad hoc environment, a connection point has the connection with all the points existing within its coverage limits when a point changes from the coverage limit, the connection deceased between the points. This leads to communication loss on broadcast. The highly active MANET causes connection loss continuously. A specific point derives out of the coverage limit of the present connection point concurrently it also establishes the connection with a fresh connection point. This feature of connection point makes this mechanism of realizing a consistent path deadly act.

To develop an energy efficient path for any source to a destination node, the EER considers the nodal energy queue occupation to generate fuzzy rules. During the path detection phase, when a source node floods a file. Route request message (RREQ) to neighboring nodes; only eligible nodes can forward this message. The central node applies the fuzzy rules described in the previous section and decides if it can contribute to build the path. The node with the lowest power cannot forward the RREQ message. Therefore, EER limits Forwarding of RREQ messages to select suitable higher power nodes. Other nodes cannot forward RREQ messages to no avail. This restriction greatly reduces network overhead. The next suitable the nodes will receive the message and forward it to the next node in the same way; Unless it's the desired destination node. This also saves grid energy and ensures greater grid longevity.

In this paper, we suggest a consistent steering mechanism EER for MANET. This steering mechanism chooses the successor points between source and destination constructed on three measures.

- The channel caliber

- Connection caliber

- Energy level of the node 


\subsection{Channel caliber}

In mobile Adhoc Networks, node-to-node channel quality varies swiftly. The variation in channel quality severely affects the QoS offered by MANET communication. The channel quality is the information about how good/bad the communication channel is. The quality of the channel exists between two nodes is measured based on Signal to Noise Ratio(SNR), Signal to Interference plus Noise Ratio (SINR) so, the information about channel quality comprises Received Signal Strength Indication (RSSI) and Bit Error Rate (BER)

The channel caliber implies that readiness of the path while communication between end points. Calculating the nosiness in the path will assistance us to apply the resource efficiently to confirm consistent routing. This parameter is applied to minimize the jitter by distribution of the resources effectively [15]. The signals are spread over the network medium. This parameter is stated as frequency at which information consistently communicated through intermediate. The channel caliber $(\mathrm{H})$ is computed by.

$$
H=G_{t} * G_{r} * \frac{\lambda}{4 \pi d}
$$

Where,

Gt Starting Point Gain,

Gr Receiving Point Gain,

$\lambda$ Positioning of Signal,

d Distance among Starting Point and Ending Point.

\subsection{Connection Caliber}

Connection caliber is valued from the value of the recipient signal. In this proposed work, a navel conection caliber measure is presented. In order to calculate the connection caliber, the time duration of connection survives between the points is taken. In the suggested mechanism, we apply the connection caliber dimension to minimize the path loss in this unpredictable situation. Though, the correct picture of wireless connections in MANETs is a deadly job. But the fresh parameter can be simply evaluated built on the transmission limit, relative speed between the connection points. The time during which the connection persists between the connection points is calculated by:-

To calculate outstanding life time of a point, foremost we have to discover the space between connection points and its relative speed. Consider a link A-B, D = Distance among points A \& B. Let (x1, y1) be the coordinates of point $\mathrm{A}$ and $(\mathrm{x} 2, \mathrm{y} 2)$ be the coordinates of point $\mathrm{B}$. Then, the remoteness is estimated by applying the formula

$$
D=\sqrt{\left(\left(X_{2}-X_{1}\right)^{2}-\left(\left(Y_{2}-Y_{1}\right)^{2}\right.\right.}
$$

- $\mathrm{R}=$ Transmission range

- The relative speed is given by the formula.

$$
\Delta V=V_{A}-V_{B}
$$

- Residual life time of the connection is

$$
T=(R-d) / \Delta V
$$

\subsection{Residual energy}

The MANET comprises of resource limited mobile nodes. The most important recourse to alive in communication is power for each node. The power is supplied to the wireless nodes using batteries. So, the efficient use of energy or power prolongs the mobile nodes.

The remaining energy level of the particular connection point is sum of existing otherwise outstanding power in particular point. The remaining energy is calculated by:-

$$
R_{E}=I_{E}-\left(N_{B} * T_{P}\right)
$$

Where,

$I_{E} \rightarrow$ Initial energy

$N_{B} \rightarrow$ No of bytes communicated

$T_{P} \rightarrow$ Broadcast power per byte 


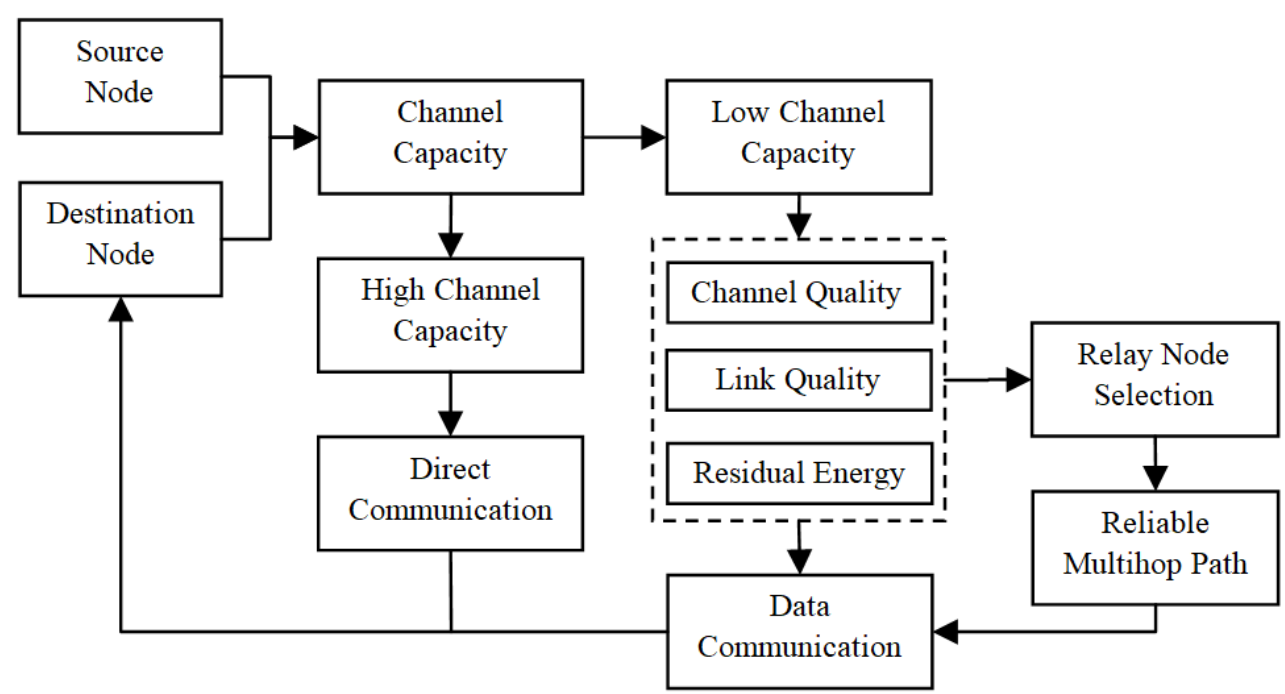

Fig.1. Proposed scheme block diagram

The blocks described in Fig. 1 to discover the reliable multi-hop path are explained briefly in the following algorithm.

\subsection{Algorithm}

The steps involved in EER routing strategy is as follows: -

- Initially, the source node checks the path survives between itself and the terminus. The Channel caliber is the degree at which the information can be unfailingly communicated over the channel.

- If the channel caliber is greater than certain brink, the source point transmits critical data directly or it will transmit the data through relay nodes. To ensure the reliable communication and energy efficiency, the source node selects the convey nodes in the below mentioned style.

- The source point transmits the Request to Relay (RTR) packets to its neighbor nodes. The RTR packet set the relay field as 1 and attaches the destination address in the header field.

- The relay node that they are nearer to the end point than the starting point node will send the Clear to Relay (CTR) packets to starting point.

- Starting point selects relay point based on the channel caliber, link residual life and the power level of mobile point and forward its data frame towards the destination.

- The source will wait for the acknowledgement packets from the end point.

- If it receives the acknowledgement container within the certain interval, it sends the next data frame or it will retransmit the last data frame.

- $\quad$ The ACK is unicasted to the source node through same path, so we can reduce the ACK overhead.

In Mobile Ad-hoc Network, the unplanned path failure is because of medium dying, path loss and lowest energy in the point canal dying roots intrusion network. Hence Quality of Service gets minimized. The connection loss in the network directs to reformation of path. The regular path renovation roots jitter and complexity. Whenever the point in the route gets left, the route to be reestablished. Hence, the suggested mechanism minimizes the path loss with the support of the parameters like channel caliber, connection caliber and remaining energy in the node. This further helps to choose the promoting point in the route.

\section{Simulation Results}

We carried out a sequence of tryouts in which the point rapidity differs 5 to $20 \mathrm{~m} / \mathrm{s}$. Points spread in the extent of $1100 \times 1100 \mathrm{~m}$. Constant Bit Rate $(\mathrm{CBR})$ flow produced. The data transmission rate aimed at passage $2 \mathrm{Mb}$ at the beginning period. Information is arranged later $0.05 \mathrm{~ms}$. Complete replication model measures mentioned in Table I. 
Table 1. Simulation Measures

\begin{tabular}{cc}
\hline \hline Parameter Type & Parameter Value \\
\hline Replication period & $20 \mathrm{~ms}$ \\
Replication space & $1500 \times 1100 \mathrm{~m}$ \\
Movement Swiftness & $5,10,15,20 \mathrm{~m} / \mathrm{ms}$ \\
Route deprivation model & Two Ray Ground \\
Canal transmission rate & $2 \mathrm{Mbps}$ \\
MAC & 802.11 \\
Communication range & $250 \mathrm{~m}$ \\
Traffic flow model & CBR \\
\hline \hline
\end{tabular}

\subsection{Simulation Results}

NS2 is Network Simulator Version 2. It is an open-source emulator intended predominantly for study in this field. To design the graph, X-graph is used. The functioning assessed by applying the measures such as packet loss ratio, Sachet transfer proportion, throughput, routing complexity and end to end interval.

The Sachet transfer proportion is the proportion of the information sachets distributed to the terminus effectively. The Packet transfer ratio is used to calculate the excellence of link. The procedure applied to discover the Proportion of packet transfer is:

$$
P D R=\frac{\text { No of packets delivered }}{\text { Time }}
$$

Fig.2(a) stretches the chart for Sachet transmission proportion. Chart displays that; projected system offers good throughput. The projects mechanism beats for several motion speediness. Fig.2 (b) gives the contrast study yield of projected mechanism using current pattern PSR. The projected mechanism gives good results than PSR. Increase in the Sachet distribution proportion specifies that it improves the behavior of the complete network.

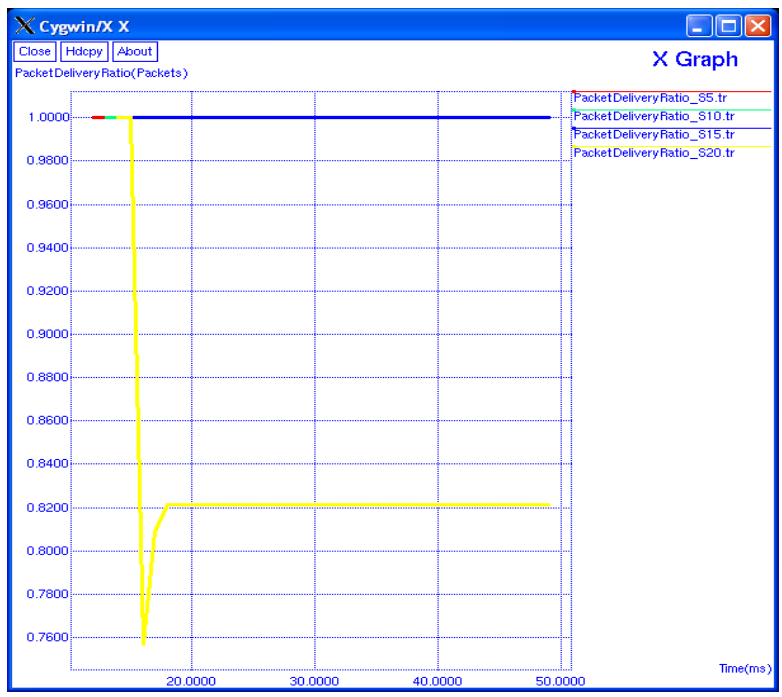

Fig.2(a). Packet Transfer Proportion investigation for many movement swiftness 


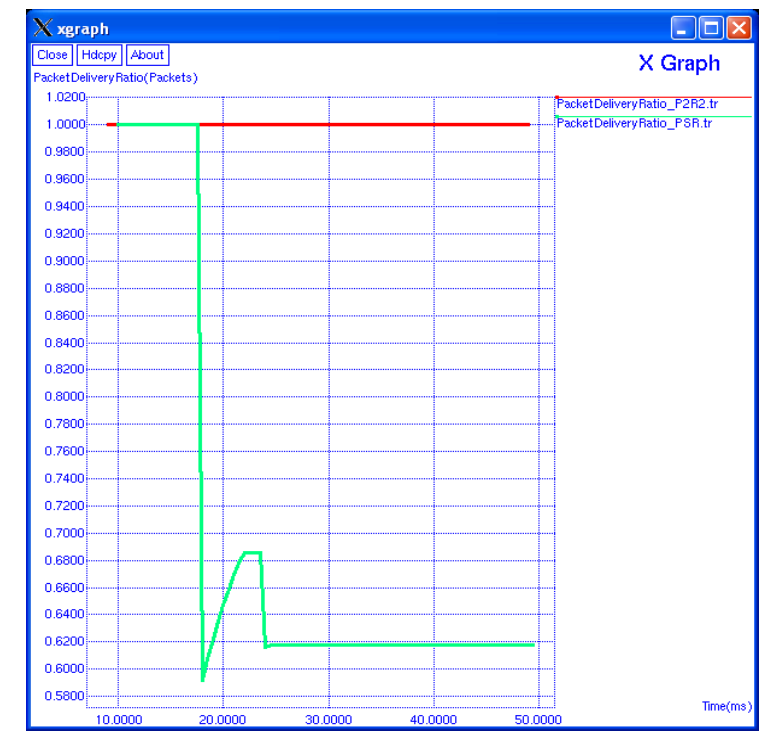

Fig.2(b). Packet Transfer Proportion evaluation study with PSR

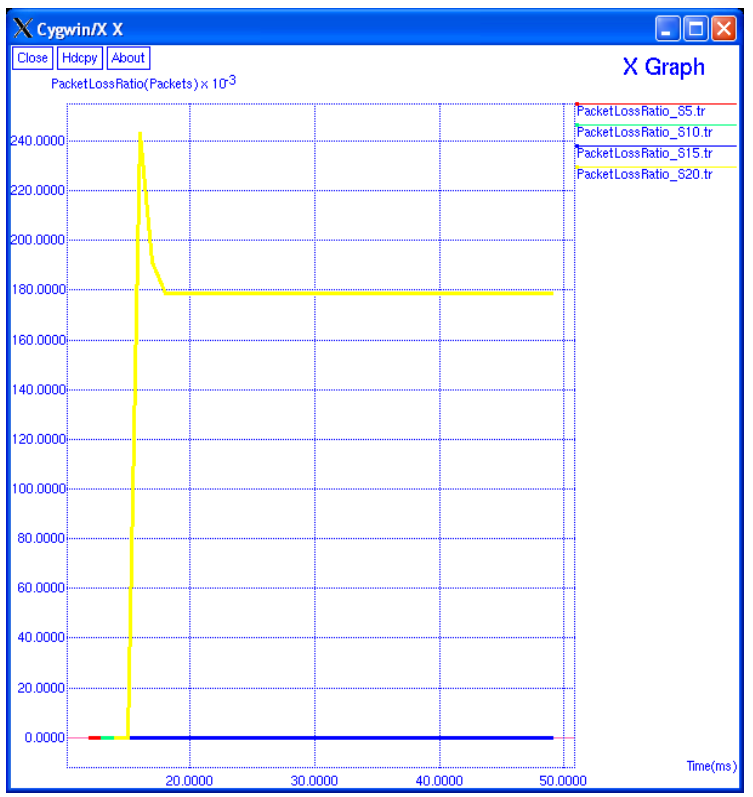

Fig.3(a). Packet Damage Proportion study for several movement swiftness

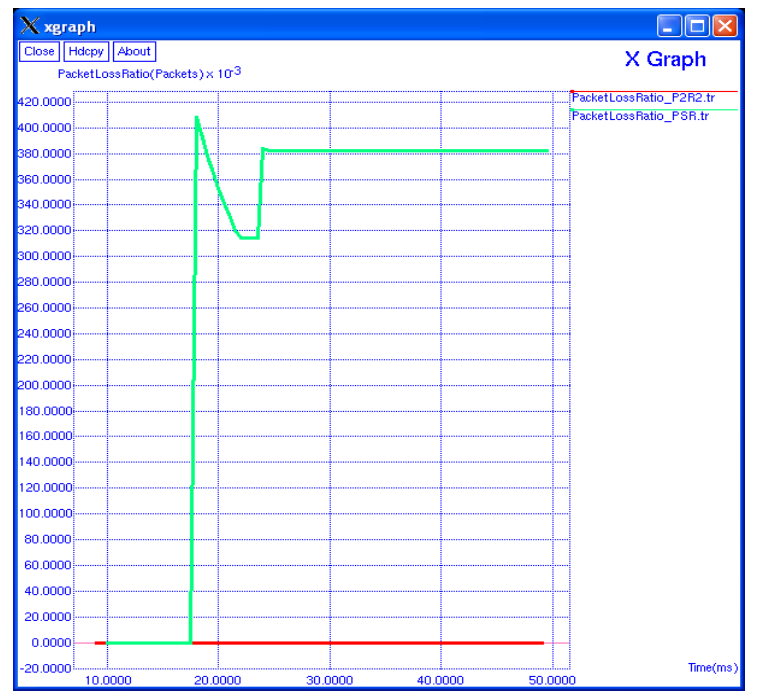

Fig.3(b). Packet Damage Proportion evaluation investigation by PSR 
The Sachet damage proportion is applied to calculate the value of the network given by the suggested mechanism. Fig. 3(a) displays the chart for Sachet damage proportion of the suggested mechanism in different replication atmosphere of dissimilar point swiftness. The Sachet damage proportion of this method is unexpected minimum value for different movements. The proposed scheme is related with the PSR methodology. PSR method shows higher than suggested scheme as shown in Fig. 3(b). Minimum in the Sachet damage proportion shows that the good performance network.

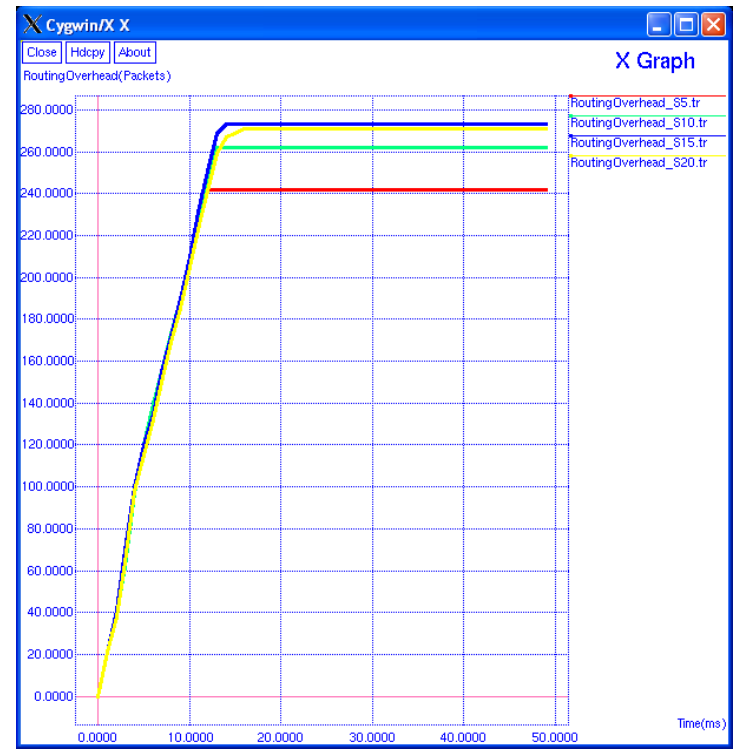

Fig.4(a). Evaluation of routing complexity for many mobility speeds

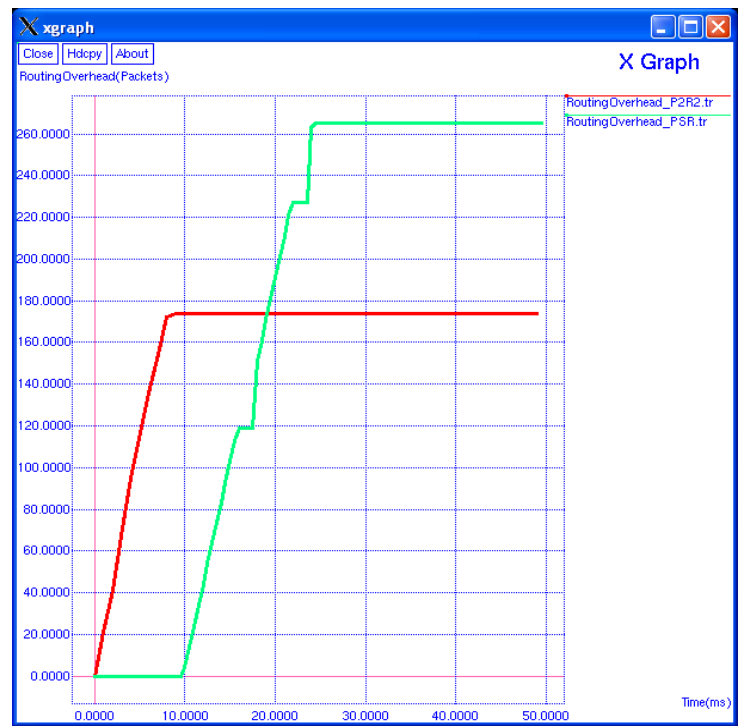

Fig.4(b). Routing complexity assessment study with PSR protocol

The communication complexity is the addition of the no. of containers communicated to observe the path to achieve the end point. The communication complexity is evaluated by the formula

$$
\text { Routing Overhead }=\sum\left(N_{R R E Q}-N_{R R E P}\right)
$$

Where,

$N_{R R E Q} \rightarrow$ No. of Routing requesting packets

$N_{R R E P} \rightarrow$ No. of Routing replying packets

The communication complexity rises whereas growing the speed of the point, then its leftovers persistent while growing the node count as displayed in Fig.4(a). The outcome revealed Fig.4(b) shows that the communication complexity in PSR is greater than the projected method as PSR develop the BFS to notice the route to each end point. 
The remaining energy (Existing Level) is evaluated by applying the formula

$$
\text { Residual Energy }=E_{r}-\left(n * P_{r}\right)
$$

Where,

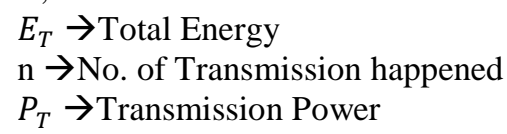

Fig.5(a) displays the projected system eats minimum energy since choosing high volume point to advance the packets near the end point. It clearly tells that the projected method gives the better energy level so that it gives good life for the battery. The evaluation study of the projected method with the current communicating method PSR is displayed in Fig. 5(b). The energy used by PSR is higher than the energy consumed by our proposed scheme. So, the energy of the various points in the network is high for the projected method once related with the current method PSR.

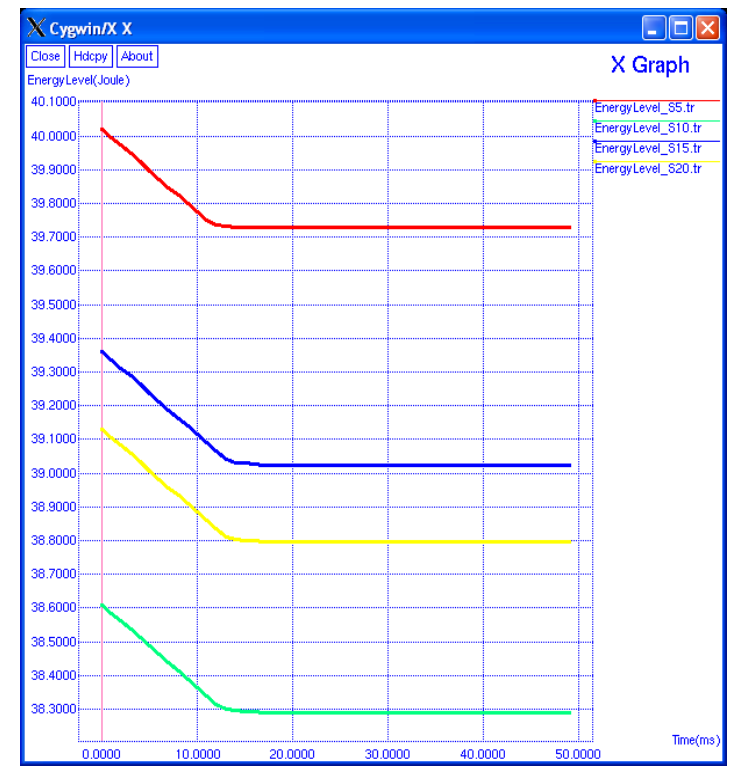

Fig.5(a). Energy level study for several movement swiftness

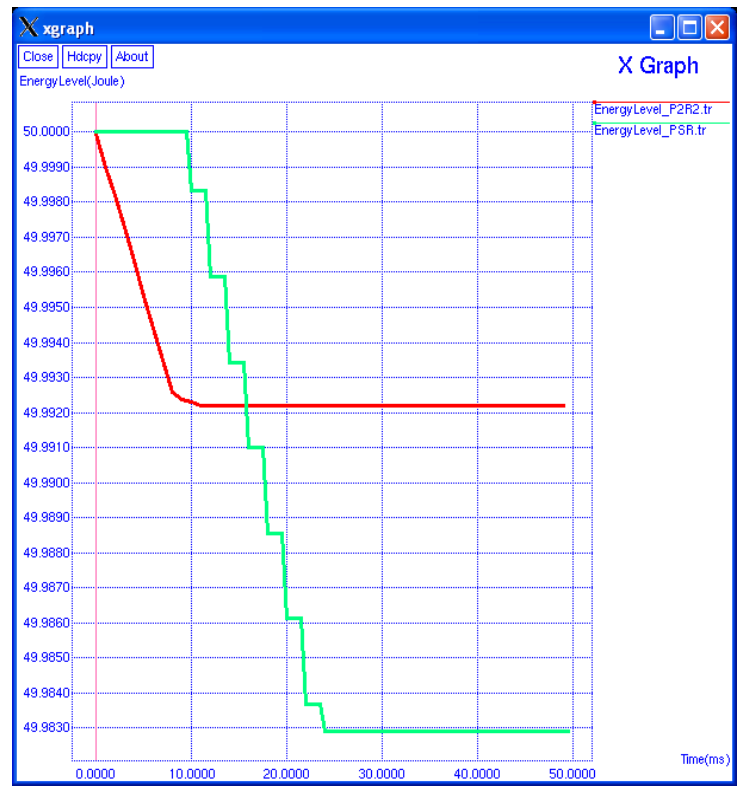

Fig.5(b). Energy level evaluation study through PSR 


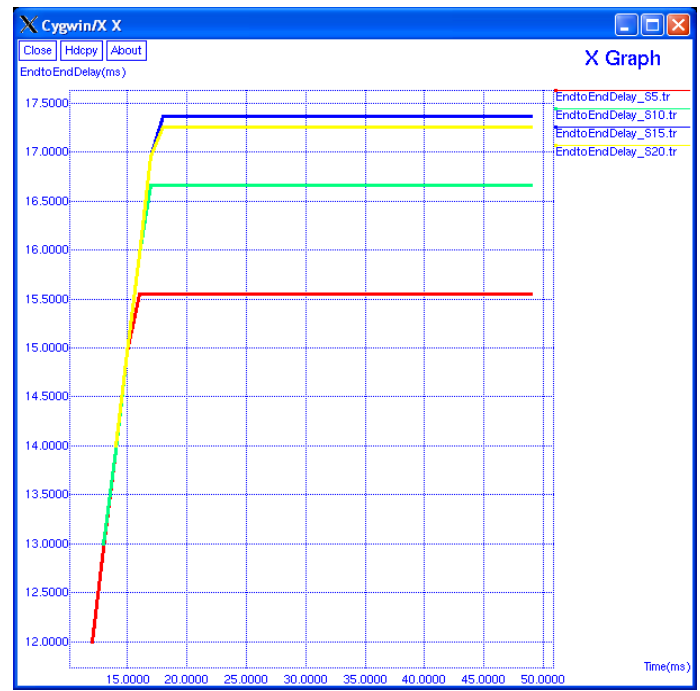

Fig.6(a). End to end delay study intended for many movement swiftness

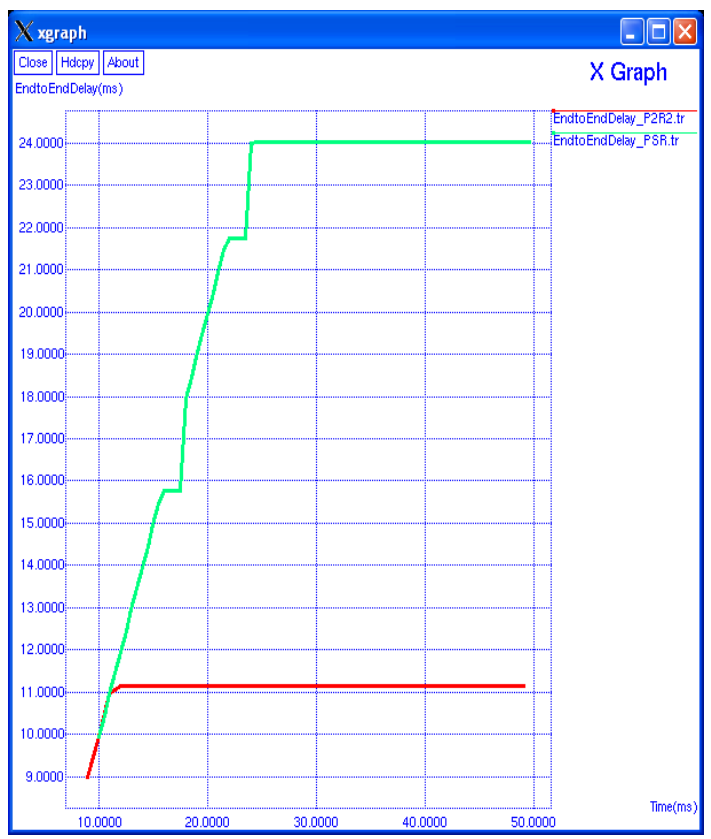

Fig.6(b). End to end delay evaluation study for PSR

The time engaged by the starting point to send the packet effectively to the end point is known as End to End delay. Procedure applied to evaluate the End to End delay is

$$
\text { End to End Delay }=A_{r}-\frac{s_{r}}{n}
$$

Where,
$A_{T} \rightarrow$ Arrival Time
$\mathrm{n} \rightarrow$ Number of Connections
$S_{T} \rightarrow$ Sent Time

Fig. 6(a) displays that the End to end study of projected method. Stay rises as going swiftness of point rises. Then mall difference will be there. The projected method directs to endured delay only in the entire network. Fig. 6(b) displays the projected routing method directs to low interval when associated through current style with PSR. 


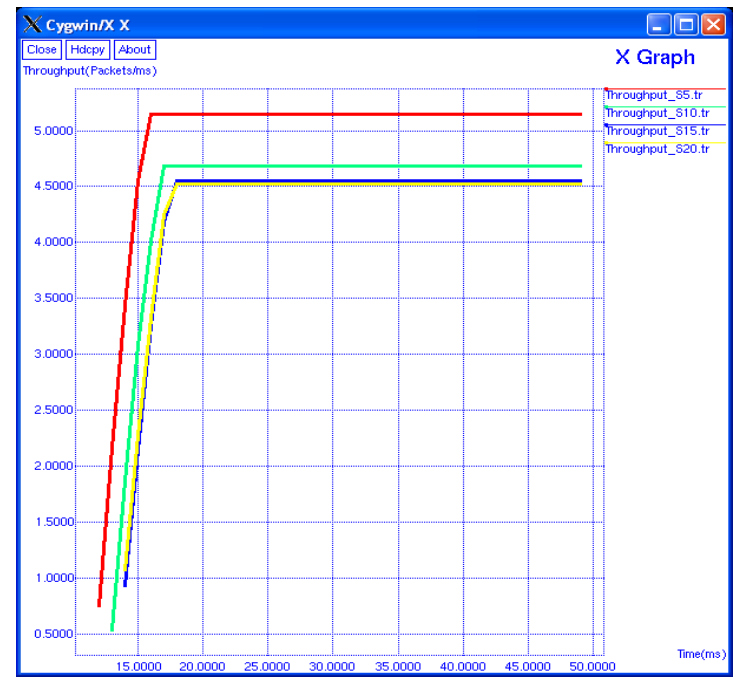

Fig.7(a). Throughput study-projected system for several movement swiftness

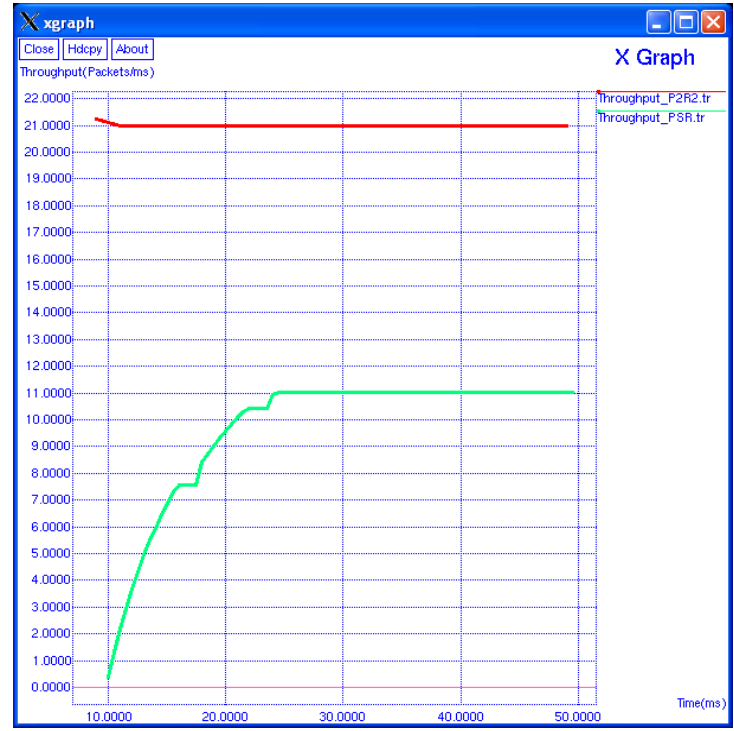

Fig.7(b). Throughput assessment study through PSR

The Throughput is evaluated with the formula

$$
\text { Throughput }=\frac{\text { No of packets delivered }}{\text { Time period }}
$$

The scheme offers good throughput whereas the point is move forward while related to the point with maximum speed as displayed in Fig. 8(a). The relative study of the projected method with current communicating strategy PSR is provided by plot displayed in Fig. 8 (b). The projected methodology can talented to assurance Quality of Service supplies in this heavy uncertain condition.

The projected system performance is evaluated by relating it with PSR scheme. The found results are given as Table II.

Table 2. Comparative Analysis

\begin{tabular}{|c|c|c|}
\hline Parameter & EER & PSR \\
\hline Output & $99.96 \%$ & $86.4757 \%$ \\
\hline Sachet Transfer Proportion & $99.78 \%$ & $69.91 \%$ \\
\hline Packet Loss Ratio & $0.24 \%$ & $26.22 \%$ \\
\hline End to End Interval & $0.778 \%$ & $11.3215 \%$ \\
\hline Transmitting Complexity & $10.561 \%$ & $34.899 \%$ \\
\hline Energy Level & $99.986 \%$ & $99.98 \%$ \\
\hline
\end{tabular}


With the help of the obtained results, the projected method yields $13.48 \%$ of throughput higher than the existing routing scheme PSR. Higher throughput indicates that the better performance of the projected method. The projected method can provide $99.78 \%$ packet delivery ratio when we configure the nodes according to the parameters specified in Table I. Mean while, the existing routing scheme yields $69.9 \%$ of sachet transfer proportion for similar network environment. From overall packets transmitted, $0.24 \%$ of the packets have dropped due the dynamic nature of the MANET for EER. However, the existing routing scheme PSR dropped $26.22 \%$ of the total transmitted packets. The projected method EER perform better for $10.53 \%$ in terms of end to end interval. Routing overhead of projected method is $24.34 \%$ lower than the existing directing method PSR. The difference between the projected and the existing scheme in terms of energy level is about $0.015 \%$. Thus, the overall performance of the projected method is much better than the existing method.

\section{Conclusion}

The system we projected in this paper is the much essential routing methodology in the highly dynamic mobile adhoc environment. This paper projects a fresh routing procedure called energy efficient routing protocol (EER). This routing mechanism applied vital measures directs to path loss in mobile ad-hoc network whereas determine path on the request. The projected scheme gives good performance even in boosting the mobility. When comparing with the existing scheme PSR, the projected scheme output showing better performance. The packet loss of the projected scheme is minimal as compared with existing scheme PSR. The results obtained by the simulation clearly show that the routing complexity in the existing PSR is more than the projected EER. It also reveals that the projected scheme is energy efficient since it is choosing reliable nodes to move the data further to reach the destination. The simulation results also show that the projected scheme supports dynamic networks by achieving low delay while comparing with existing PSR. Channel Caliber, connection caliber and Remaining Energy level are the three vital measures we are taken in to account. Proper inspection of remaining energy of the path between the ends, minimizes the path loss while communication. Examination of Connection Caliber while path finding procedure rises the complete throughput. Energy remained while forwarding exercise will decrease the end system failure during communication. All results of simulation reveal that, projected mechanism gives improved show for several mobility speed.

\section{Acknowledgements}

I would like to thank my supervisor, Professor Dr. N. Sankar Ram for his continuous encouragement and support. I also like to thank all my co-authors and my colleague who helped me out in completing this paper.

\section{References}

[1] Karim El Defrawy, Gene Tsudik, "Privacy-Preserving Location-Based On-Demand Routing in MANETs", IEEE, December 2011.

[2] Ming Yu, Mengchu Zhou and Wei Su, "A Secure Routing Protocol against Byzantine Attacks for MANETs in Adversarial Environments", IEEE, April 2008.

[3] Kiran Manjappa, Ram Mohana Reddy Guddeti, "Mobility aware-termite: a novel bio inspired routing protocol for mobile adhoc networks", IET Networks, December 2013.

[4] Sunho Lim, Chansu Yu; Chita R. Das, "RandomCast: An Energy-Efficient Communication Scheme for Mobile Ad Hoc Networks", IEEE, January 2009.

[5] Shivashankar Golla Varaprasad,Suresh Hosahalli Narayanagowda, "Implementing a new power aware routing algorithm based on existing dynamic source routing protocol for mobile ad hoc networks", IEEE, June 2014.

[6] Cong Liu, Jie Wu, "Virtual-Force-Based Geometric Routing Protocol in MANETs", IEEE, June 2008.

[7] Zehua Wang, Yuanzhu Chen; Cheng Li, "PSR: A Lightweight Proactive Source Routing Protocol For Mobile Ad Hoc Networks", IEEE, August 2013.

[8] Lavanya. Poluboyina, Sivakumar Reddy. V, Mallikarjuna Prasad. A, "Evaluation of QoS Support of AODV and its Multicast Extension for Multimedia over MANETs", International Journal of Computer Network and Information Security (IJCNIS), Vol.12, No.1, pp.13-19, 2020. DOI: 10.5815/ijcnis.2020.01.02

[9] Ritu Sharma, "A Secure and Proficient Routing Protocol in Mobile Ad-hoc Networks using Genetic Mechanism", IJIRCCE, June 2016

[10] A.Manikandan, S.Pradeep, "Quantitative Analysis of Network Arrangement in Randomized Appropriation in WSN" Journal of Chemical and Pharmaceutical Sciences, pp 181-184, 2017.

[11] Michal Pióro,Dritan NaceYoann and Fouquet, "On Protected Traffic Routing in Wireless Networks with Partial Multiple Link Failures", IEEE Computer Society Washington, 2013.

[12] Manikandan, A., \& Rajarajachozhan, C. (2017). Artificial Bee Colony for Socially Aware Networking. Journal of Chemical and Pharmaceutical Sciences, 2, 299-301.

[13] Jihui Zhang,Qian Zhang,Bo Li,Xiaonan Luo, "Energy-efficient routing in mobile ad hoc networks: mobility-assisted case", IEEE, Jan 2016.

[14] Mahaboob Sharief Shaik, Fahad Mira, " A Comprehensive Mechanism of MANET Network Layer Based Security Attack Prevention ", International Journal of Wireless and Microwave Technologies (IJWMT), Vol.10, No.1, pp. 38-47, 2020.DOI: 
10.5815/ijwmt.2020.01.04

[15] Mohammed Azza, Sofiane Boukli Hacene,"An Enhanced Reputation-based for Detecting Misbehaving Nodes in MANET", International Journal of Wireless and Microwave Technologies (IJWMT), Vol.7, No.4, pp.28-37, 2017.DOI: 10.5815/ijwmt.2017.04.03

[16] Tanweer Alam, Baha Rababah, "Convergence of MANET in Communication among Smart Devices in IoT", International Journal of Wireless and Microwave Technologies (IJWMT), Vol.9, No.2, pp. 1-10, 2019.DOI: 10.5815/ijwmt.2019.02.01

[17] Salem Satia, Ahmed El-bareg," MANET Testbed using Raspberry PIs", International Journal of Wireless and Microwave Technologies (IJWMT), Vol.8, No.2, pp. 52-63, 2018.DOI: 10.5815/ijwmt.2018.02.05

[18] Rafi U Zaman, S. Shehnaz Begum, Khaleel Ur Rahman Khan, A. Venugopal Reddy,"Efficient Adaptive Path Load Balanced Gateway Management Strategies for Integrating MANET and the Internet", International Journal of Wireless and Microwave Technologies (IJWMT), Vol.7, No.2, pp.57-75, 2017.DOI: 10.5815/ijwmt.2017.02.06

\section{Authors' Profiles}

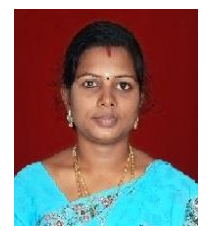

Dr. D. S. JAYALAKSHMI received her Bachelor's degree in Physics in 2004 from Bharathi Women's College, University of Madras, Master's degree in Physics in 2006 from Queen Mary's College, University of Madras and Master of Philosophy in Physics in 2007 from Queen Mary's College, University of Madras. She completed her Ph.D., in Sathyabama University, Chennai in 2014. She has been working on the computation of the electronic band structure calculation. She has published nearly 20 reputed journals. She has keen interest in the study of condensed matter physics; Structural phase transition, bonding properties, high pressure studies, superconductivity studies and Fermi surface analysis are her fields of interest.

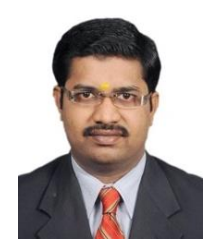

Dr. D. Hemanand is currently working as an Assistant Professor of Computer Science and Engineering at Sriram Engineering College, Tamilnadu. India. He is dedicated to realize the vision and mission of the Sriram Educational Trust. He received his B.E. degree in Electronics and Communication Engineering from Sriram Engineering College, Madras University, Chennai and M.E. in Computer Science and Engineering from Velammal Engineering College, Anna University, Chennai and Ph.D. in Information and Communication Engineering from Anna University, Chennai. He has 14 years of vast teaching and research experience and he has published more than 25 papers in National/International journals and conferences. His primary research interests include Computer Networks, Network Security, Mobile Computing etc.

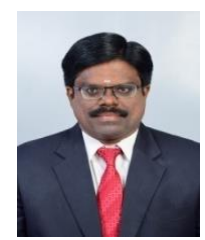

G. Muthu kumar, currently working as an Assistant professor in Computer science and Engineering department in Sriram Engineering college, Tamilnadu. India. He has 17.0 years of experience in the teaching field in various Engineering college. I completed my UG degree in computer science and engineering from Sankara university and PG degree from St. Peter's university. He has published many papers in various national and international conferences all over India.

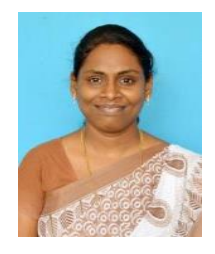

M. Madhu Rani, She is currently working as an Assistant Professor in Computer science and Engineering department in Rajalakshmi Engineering College, Tamilnadu. India. She has 13 years of teaching experience and in Computer Science and Engineering at reputed engineering colleges in Tamil Nadu. I am confident that I would live up to your institute's expectation with this strong background in teaching. She received her B.E., degree in Computer Science and Engineering from Dr.G.U.POPE College of Engineering, Affiliated to Anna University and M.E., degree in Computer Science and Engineering from Sathyabama University, Tamilnadu, India. She has published many papers in various national and international conferences.

How to cite this paper: D. S. Jayalakshmi, D. Hemanand, G. Muthu Kumar, M. Madhu Rani, "An Efficient Route Failure Detection Mechanism with Energy Efficient Routing (EER) Protocol in MANET", International Journal of Computer Network and Information Security(IJCNIS), Vol.13, No.2, pp.16-28, 2021. DOI: 10.5815/ijcnis.2021.02.02 